\title{
L'ARPKD in 4 sessioni e una fiaba illustrata
}

\author{
Catia Cirulli, una attiva volontaria di AIRP onlus, ha partecipato all'incontro "ARPKD chiama, AIRP risponde", \\ che si è tenuto lo scorso 27 settembre a Roma e ne ha tratto questo resoconto dettagliato, utile sia per chi non \\ era presente, sia per chi, invece, c'era \\ e può ritrovare qui tutti i contenuti principali del seminario. Ricordiamo che l'incontro di Roma è stato fortemente \\ voluto da AIRP, che da anni si occupa della forma dominante di questa malattia, ma, da quest'anno, ha esteso \\ il proprio impegno anche alla forma recessiva. Da qui, l'idea di organizzare un seminario per dare risposte \\ alle famiglie, ma anche per aggiornare la comunità scientifica sulle più recenti evidenze in tema
} di rene policistico autosomico recessivo. Ecco il resoconto di Catia. (rev. scientifica: Dr.ssa Laura Massella)

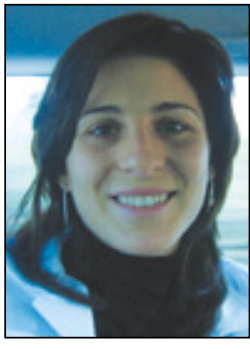

Catia Cirulli
Il simposio inizia con i saluti dei Responsabili Scientifici, in particolare della Dr.ssa Laura Massella, dirigente medico presso l'U.O. di Nefrologia e Dialisi dell'Ospedale Bambino Gesù, e del Prof. Francesco Scolari, Direttore di U.O.C. di Nefrologia e Dialisi presso gli Spedali Civili di Brescia, nonché Professore Associato all'Università di Brescia.

Lo spirito dell'incontro è il desiderio di condividere progetti e idee per avvicinare i medici ai genitori, che tante volte si trovano disorientati di fronte a questa malattia, ma che dimostrano sempre molto coraggio nell'affrontarla (Fig. 1).

Seguono brevi interventi di saluto anche da parte del Dr. Andrea Bartuli, Direttore di U.O. delle Malattie Rare dell'Ospedale Bambino Gesù, e di Luisa Sternfeld Pavia, Presidente di AIRP onlus - Associazione Italiana Rene Policistico.

La prima sessione del convegno è dedicata alla genetica e alla biochimica della malattia, moderata dal Prof. Francesco Emma, responsabile dell'U.O. di Nefrologia e Dialisi presso 1'Ospedale Bambino Gesù, e dalla Dr.ssa Alessandra Boletta, ricercatrice presso la Divisione di Genetica e Biologia Cellulare dell'Ospedale San Raffaele di Milano.

Il primo relatore è il Dr. Salvatore Melchionda dell'Ospedale Casa Sollievo di San Giovanni Rotondo, che spiega subito come la malattia si trasmetta in modo autosomico recessivo, cioè dall'incontro di due genitori, che hanno nel loro patrimonio genetico un allele sano e uno mutato; in loro, la malattia non si manifesta, ma dalla loro unione, si ha un $25 \%$ di probabilità di avere un figlio affetto, che eredita cioè un allele malato dalla madre e uno dal padre, un altro $25 \%$ dei figli saranno sani e un $50 \%$ portatori sani. Questo calcolo delle probabilità si ripete a ogni gravidanza.

Il Dr. Melchionda prosegue, poi, con l'inquadramento genetico della malattia policistica recessiva, specificando che essa è causata da un'ampia gamma di mutazioni del gene chiamato PKHD1, posizionato sul cromosoma 6, formato da 86 esoni, che codifica per una proteina, chiamata fibrocisti- na. Si stima che i portatori sani nella popolazione generale siano 1/70 e che l'incidenza di malattia sia 1:20 000. Viene spiegato ancora che attualmente la ricerca mutazionale viene effettuata con la nuova tecnica di "Next Generation Sequencing" (NGS), che ha permesso di abbreviare notevolmente $\mathrm{i}$ tempi di attesa.

L'argomento viene ripreso, di seguito, dal Dr. Gianfranco Savoldi, biologo presso gli Spedali Civili di Brescia, che presenta nel dettaglio la tecnica del "Next Generation Sequencing" e la confronta con la tecnica attualmente in uso, il "Sanger Sequencing". Questo metodo impone, infatti, tempi di attesa lunghi, giacché l'analisi viene svolta in maniera sequenziale (il primo esone poi il secondo poi il terzo e così via), mentre con l'NGS si analizzano tutti gli esoni simultaneamente e in modo automatico. Presso il loro laboratorio, sono a punto sia il gene della fibrocistina sia il gene PKD2 (uno dei due geni coinvolti nella forma dominante), ma anche altri geni coinvolti in diverse forme di ciliopatie più rare. Con l'NGS i tempi di refertazione arriveranno a ridursi fino a sei-otto settimane.

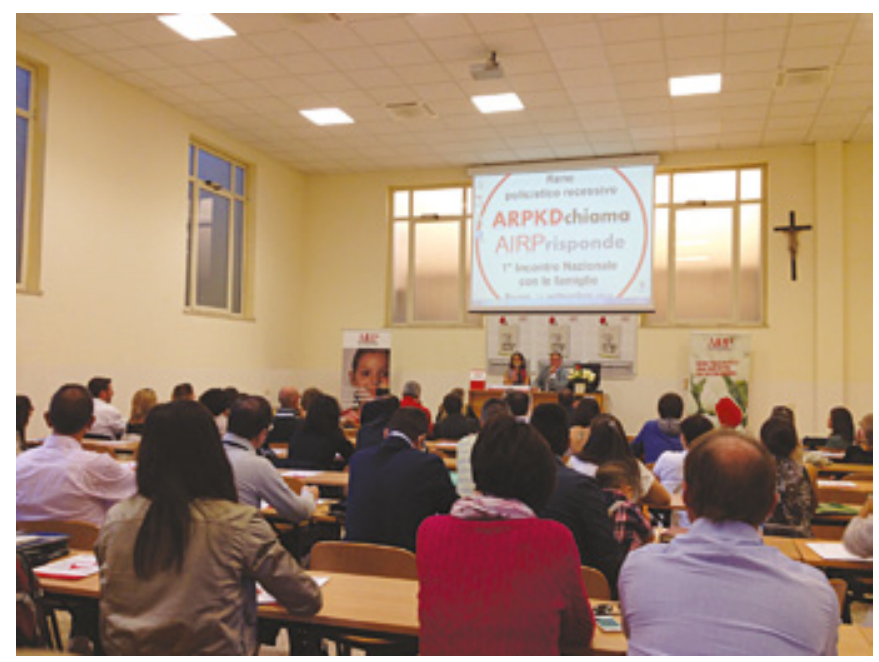

Fig. 1 - Vista dei partecipanti. 


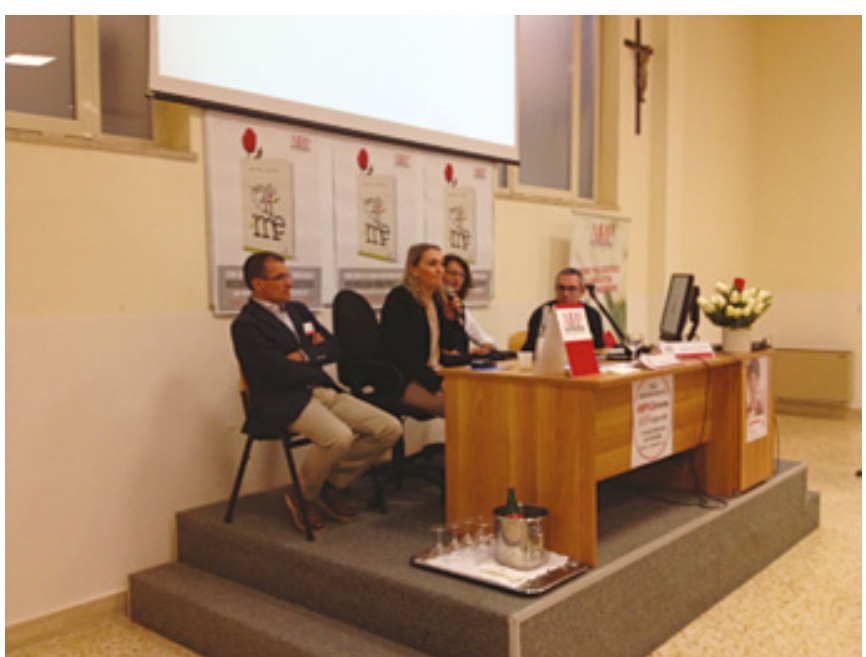

Fig. 2 - Da sinistra: Salvatore Melchionda, Claudia Izzi, Alessandra Boletta, Gianfranco Savoldi.

Segue la Dr.ssa Claudia Izzi, dirigente medico della U.O. di Diagnosi Prenatale, Dipartimento Ostetrico-Ginecologico e Divisione di Nefrologia, Spedali Civili di Brescia, che spiega il punto di vista del genetista clinico in epoca prenatale. La Dottoressa spiega l'importanza del raccogliere una buona e dettagliata anamnesi familiare, parla dell'approccio ecografico in epoca prenatale e della difficoltà nel distinguere tra una forma dominante e una recessiva di policistosi renale, quando la familiarità non è chiara e non orienta nell'uno o nell'altro senso. Un'altra difficoltà è data dal fatto che, soprattutto in epoca prenatale non sempre la malattia è evidente in entrambi gli organi e questo aggiunge ancora qualche dubbio all'orientamento diagnostico. E chiaro che, nel caso in cui sia disponibile un test genetico diagnostico effettuato in precedenza su un altro familiare con mutazione identificata, allora è possibile effettuare una diagnosi prenatale di certezza con villocentesi tra la decima e l'undicesima settimana di gestazione.

Da ultimo, ascoltiamo la Dr.ssa Alessandra Boletta, ricercatrice di livello internazionale e anche moderatrice nella stessa sessione, che presenta il progetto di ricerca sostenuto da $A I R P$ e i brillanti risultati ottenuti fino ad ora. Questo progetto di ricerca è, al momento, incentrato sul rene policistico dominante e sui meccanismi attraverso i quali le cellule del tubulo renale producono energia per la crescita cellulare. I topi usati in laboratorio con mutazione di PKD1 presentano una abnorme proliferazione cellulare, che porta alla formazione delle cisti tipiche della malattia. Fornendo a questi topi malati un glucosio modificato (2DG), quindi difficilmente utilizzabile a livello metabolico come fonte di energia, si blocca la proliferazione cellulare, con netta riduzione della formazione delle cisti e, quindi, dell'aumento abnorme del volume dei reni. Si sta cercando di capire se lo stesso modello possa essere in qualche modo trasferito al rene policistico recessivo (Fig. 2).

Terminata la sessione di genetica e biochimica, si passa alla seconda sessione, dedicata agli aspetti clinici della malattia policistica recessiva, moderata dal Dr. Giovanni Montini, responsabile dell'incarico professionale di Alta Specializzazione, programma di Nefrologia Pediatrica, Azienda OspedalieroUniversitaria di Bologna, Policlinico S. Orsola-Malpighi, e del Prof. Francesco Scolari, già presentato prima.

Si discute di manifestazioni renali del paziente con ARPKD con la Dr.ssa Laura Massella. Vengono inizialmente elencate le caratteristiche generali di questa malattia: la possibilità di oligoidramnios nell'anamnesi gestazionale, la probabilità di morte in epoca neonatale pari al $25-30 \%$ circa per insufficienza respiratoria e renale insieme, la caratteristica precocità delle manifestazioni cliniche (seppure non la regola), il fatto che quando la malattia si manifesta in epoca più tardiva, il coinvolgimento epatico è prevalente rispetto a quello renale; inoltre, viene ricordato come in alcuni casi la forma di rene policistico dominante ad esordio neonatale (2-5\% dei casi) sia difficilmente indistinguibile, ad un primo approccio, dalla forma recessiva. La Dr.ssa Massella prosegue nell'illustrare i segni principali della malattia: reni grandi e iperecogeni, ipertensione, insufficienza renale, infezioni delle vie urinarie, iponatriemia nei bambini più piccoli. Secondo i dati riportati dalla letteratura, chi supera il primo anno di vita, ha un $86 \%$ di probabilità di avere una funzionalità renale normale a cinque anni e un $42 \%$ a venti anni. Nonostante la gestione di questi pazienti sia notevolmente migliorata negli anni (diagnosi precoce, maggiore attenzione all'alimentazione, al controllo pressorio), mancano, tuttavia, ancora linee guida comuni e chiare sul trattamento e la gestione assistenziale di questi pazienti. A tale scopo è in atto uno studio europeo esclusivamente sulla forma recessiva, e una raccolta dati afferente alla Società Italiana di Nefrologia Pediatrica (in questo caso sia per la forma dominante che recessiva), allo scopo di definire meglio la malattia e le modalità per una gestione ottimale.

Delle manifestazioni epatiche parla il Dr. Andrea Pietrobbattista, dirigente medico dell'U.O. di Epatogastroenterologia e Nutrizione dell'Ospedale Bambino Gesù, che si sofferma sulla malattia epatica, chiamata fibrosi epatica congenita, caratterizzata da un aumento del tessuto connettivo fibroso a carico del fegato. Spesso e molto a lungo, gli esami di funzionalità epatica sono normali, ma il fegato è di dimensioni più grandi e di consistenza aumentata per via della fibrosi; proprio questo stato di fibrosi, non permettendo più un adeguato scarico venoso di sangue dalla periferia verso il cuore, crea uno stato di ipertensione portale con milza grande fino alla formazione di varici esofagee nelle forme più gravi. L'esordio/scoperta della malattia è più frequente tra $\mathrm{i}$ cinque e $\mathrm{i}$ tredici anni, associata ad anomalie dei dotti biliari, che possono complicarsi con quadri infettivi, talvolta molto gravi, chiamati colangiti. Talvolta possono comparire cisti connesse all'albero biliare (malattia di Caroli).

Altro aspetto clinico importantissimo è quello della ipertensione arteriosa, trattato dal Dr. Marcello Chinali, dirigente medico dell'U.O. di Cardiologia presso l'Ospedale Bambino Gesù. Tra i primi e più frequenti sintomi della policistosi recessiva, infatti, c'è proprio l'ipertensione arteriosa, che, se non curata in modo adeguato può provocare danni al cuore. Nei pazienti affetti da malattia renale, spesso la morte interviene 


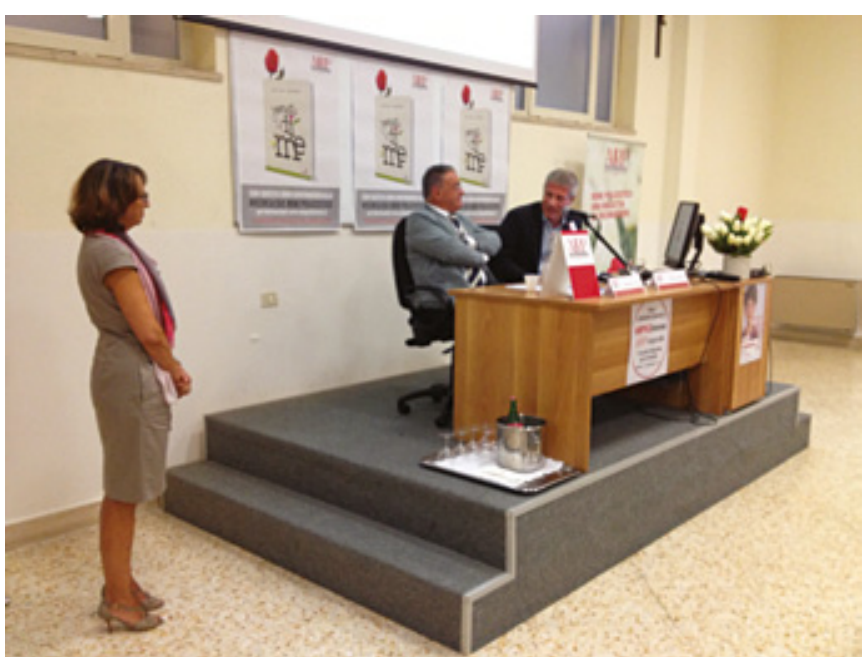

Fig. 3 - da sinistra: Laura Massella, Francesco Scolari, Giovanni Montini.

proprio per problemi di tipo cardiovascolare; da qui l'importanza di ottenere un controllo pressorio efficace fin dai primi segni di ipertensione per evitare l'insorgenza di ipertrofia cardiaca, danno d'organo secondario all'ipertensione. Viene fatto anche un breve excursus sui farmaci antipertensivi in uso nel bambino (Fig. 3)

La terza sessione del convegno è dedicata alla dialisi e al trapianto in ARPKD, moderatori il Dr. Stefano Picca, responsabile della Dialisi pediatrica presso l'Ospedale Bambino Gesù, e il Prof. Giuliano Torre, responsabile dell'U.O. di Epato-gastroenterologia e Nutrizione dell'Ospedale Bambino Gesù.

Si parla di emodialisi e dialisi peritoneale in età pediatrica con il Dr. Alberto Edefonti, direttore dell'U.O. di Nefrologia e Dialisi dell'Ospedale De Marchi di Milano, che illustra le due metodiche dialitiche, i pro e i contro nello scegliere l'una piuttosto che 1'altra. In questo contesto, il Dr. Edefonti inserisce anche il problema della nefrectomia uni- o bilaterale prima dell'inizio del trattamento dialitico.

La Dr.ssa Manila Candusso, dirigente medico dell'U.O. di Epato-gastroenterologia e Nutrizione dell'Ospedale Bambino Gesù, parla del trapianto singolo e combinato rene-fegato. Spiega come ci siano molte differenze nei pazienti con ARPKD: in alcuni la compromissione di entrambi gli organi è evidente e la decisione su un trapianto combinato è più semplice. Talvolta, però, ci si trova di fronte a una forte compromissione epatica rispetto a quella renale e viceversa: in queste situazioni, ogni bambino merita una discussione e una decisione a sé.

In alcuni casi, può essere necessario effettuare un trapianto combinato, anche se la funzione di uno dei due organi non è completamente compromessa (Fig. 4).

La quarta sessione consiste in una tavola rotonda aperta ai pazienti e alle loro famiglie. Qui le famiglie sono davvero protagoniste e raccontano le loro storie, il loro coraggio e la storia della loro malattia: chi ha avuto i primi sintomi da piccolissimo, chi è ancora asintomatico, chi ha forme particolari di rene policistico recessivo all'interno di quadri

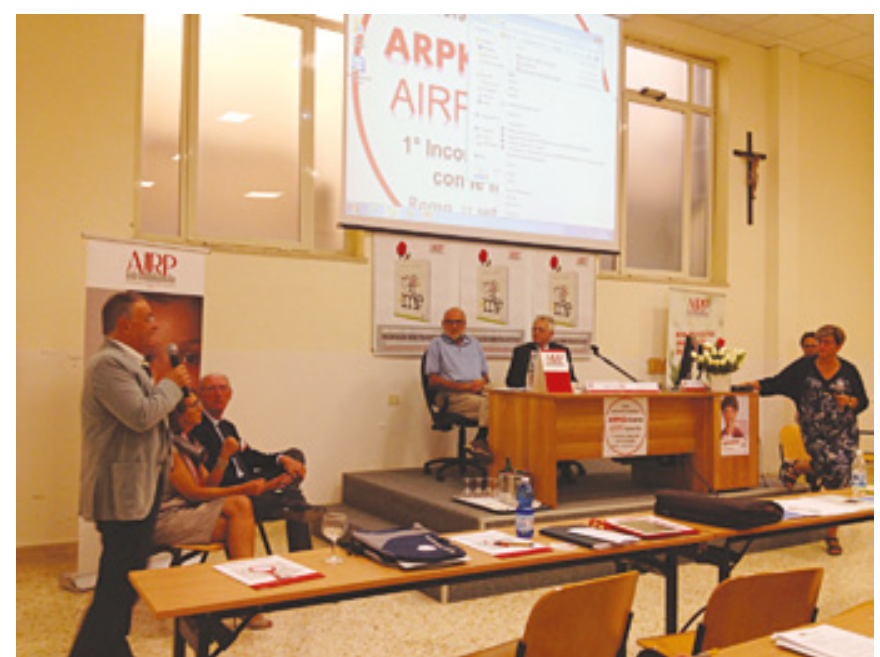

Fig. 4 - I Relatori e i Moderatori alla Tavola Rotonda.

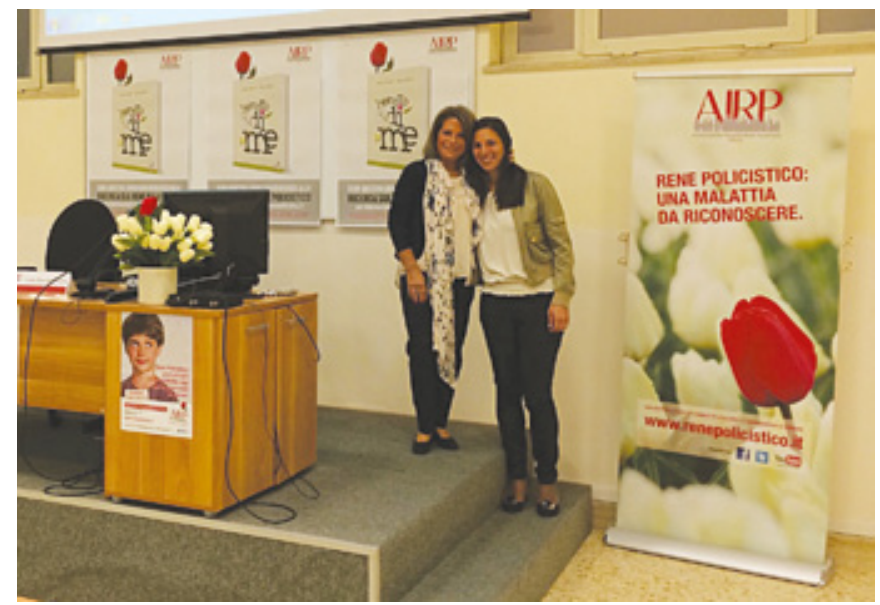

Fig. 5 - Luisa Sternfeld e Catia Cirulli.

malformativi complessi, chi ha evoluzioni così tardive da rendere molto animata la discussione dal punto di vista della diagnosi.

Nel corso del simposio, viene riservato un ampio spazio da parte di Luisa Sternfeld per presentare il libro Dentro di me, il primo gesto concreto che $A I R P$ dedica ai più piccoli, espressione di un chiaro e forte segnale di aiuto, un gesto che invita a riflettere su temi difficili come essere portatori di una malattia così impegnativa, in modo garbato e poetico, con un linguaggio semplice e tuttavia pieno di immaginazione. Il libro viene introdotto dall'autrice Cosetta Zanotti e dalla illustratrice Elena Gallina.

Cosetta Zanotti spiega come si possa parlare in molti modi della malattia, anche a dei bambini, e nello stesso tempo raccontare quanto ogni persona sia speciale.

Dentro di me racconta ad ognuno di noi, grande o piccolo che sia, che c'è una meravigliosa unicità, fatta di tante cose belle, che talvolta si mescolano a un qualcosa di meno gradito che ci rende più fragili, ma l'insieme che ne risulta è sempre unico 
bello e colorato. È proprio quello che scopre Gugù, il protagonista di questo piccolo albo, e con lui lo scoprirà ogni lettore che sfoglierà le sue pagine.

In questo convegno, dedicato allo studio scientifico della malattia, torna utile la riflessione del grande scultore Giuseppe Rivadossi: "La scienza è conoscenza, l'arte è riconoscenza”. Mentre la scienza ci permette di procedere nella ricerca e di scoprire sempre nuove soluzioni, l'arte ci indica come spalancare gli occhi dell'anima per essere riconoscenti alla vita, di ri-conoscerne la bellezza per donarla agli altri nelle forme più diverse. L'arte ci spalanca la verità della vita, anche quando essa ci chiede di percorrere sentieri che non vorremmo calpestare o di custodire amori non programmati, che non avremmo mai pensato di incontrare, accettando così il rischio di vedere aumentata "la superficie di feribilità del nostro cuore!” (Giusi Quarenghi). L'arte ci insegna a non aver paura e ad "entrare con fiducia nel mistero dell'esisten$z a "$ (Eric E. Schmitt).
Con il ricavato di questo libro - regalo natalizio ideale AIRP finanzierà, dal prossimo anno, la ricerca anche su ARPKD.

La giornata di lavori si conclude così con molte speranze da parte di tutti e con il ringraziamento a tutti i medici intervenuti e soprattutto a Luisa ed Anna Sternfeld e a Grazia Sandrini.

AIRP ha saputo ascoltare ancora una volta la voce delle famiglie, organizzando per loro una giornata ricca di emozioni. Queste famiglie, fino ad ora, si sentivano sole, ma ora non è più così.

E tutto grazie alla forza e alla volontà di una donna piccola ma molto grande.

Grazie Luisa, da parte di tutte le famiglie (Fig. 5).

Catia Cirulli 